\title{
Immunotherapy for Patients with Advanced Urothelial Cancer: Current Evidence and Future Perspectives
}

\author{
Clizia Zichi, ${ }^{1}$ Marcello Tucci, ${ }^{1}$ Gianmarco Leone, ${ }^{1}$ Consuelo Buttigliero, ${ }^{1}$ \\ Francesca Vignani, ${ }^{2}$ Daniele Pignataro, ${ }^{1}$ Giorgio V. Scagliotti, ${ }^{1}$ and Massimo Di Maio ${ }^{2}$ \\ ${ }^{1}$ Department of Oncology, University of Turin, Division of Medical Oncology, San Luigi Gonzaga Hospital, \\ Regione Gonzole 10, Orbassano, 10043 Turin, Italy \\ ${ }^{2}$ Department of Oncology, University of Turin, Division of Medical Oncology, Ordine Mauriziano Hospital, \\ Via Magellano 1, 10028 Turin, Italy \\ Correspondence should be addressed to Massimo Di Maio; massimo.dimaio@unito.it
}

Received 14 April 2017; Accepted 7 May 2017; Published 7 June 2017

Academic Editor: Carmen Criscitiello

Copyright $\odot 2017$ Clizia Zichi et al. This is an open access article distributed under the Creative Commons Attribution License, which permits unrestricted use, distribution, and reproduction in any medium, provided the original work is properly cited.

\begin{abstract}
In recent years, immunotherapy has produced encouraging results in a rapidly increasing number of solid tumors. The responsiveness of bladder cancer to immunotherapy was first established in nonmuscle invasive disease in 1976 with intravesical instillations of bacillus Calmette-Guérin (BCG). Very recently immune checkpoint inhibitors demonstrated good activity and significant efficacy in metastatic disease. In particular the best results were obtained with programmed death-ligand-1 (PD-L1) and programmed death-1 (PD-1) inhibitors, but many other immune checkpoint inhibitors, including anti-cytotoxic T-lymphocyteassociated protein-4 (CTLA-4) antibodies, are currently under investigation in several trials. Simultaneously other therapeutic strategies which recruit an adaptive immune response against tumoral antigens or employ externally manipulated tumor infiltrating lymphocytes might change the natural history of bladder cancer in the near future. This review describes the rationale for the use of immunotherapy in bladder cancer and discusses recent and ongoing clinical trials with checkpoint inhibitors and other novel immunotherapy agents.
\end{abstract}

\section{Introduction}

As well documented by a large body of research, tumor cells are able to avoid control and destruction by the immune system using a range of complex and often overlapping mechanisms that lead to disruption of key components involved in the effective antitumor response [1-4]. Immune system should recognize and eliminate tumor cells that can avoid this immune response by disrupting antigen presentation, either through downregulation of major histocompatibility complex (MHC) class I molecules or by disabling antigenprocessing machinery. Alternatively, or in addition, tumors can be able to suppress the immune system by a disruption of molecular pathways involved in controlling T-cell inhibition and activation or by recruiting immunosuppressive cell types, such as regulatory T-cells (Tregs) and myeloid-derived suppressor cells. Another mechanism that tumor cells may use in order to suppress immune activity is the release of factors, including adenosine and prostaglandin E2 and the enzyme indoleamine 2,3-dioxygenase (IDO) [3].

The robust progress in the understanding of these tumor immune-evasion strategies has resulted in the evaluation of various approaches to target and harness the patient's immune system directly to kill tumor cells. Consequently, in recent years, new generation of immunotherapy has produced relevant results in a rapidly increasing number of solid tumors. With the exception of the therapeutic vaccine sipuleucel-T that was approved for the treatment of prostate cancer in 2010, all these practice-changing results have been obtained with immune checkpoint inhibitors. Two major classes of drugs have been tested: anti-cytotoxic Tlymphocyte-associated protein (CTLA)-4 antibodies and anti-programmed death-1 (PD-1) or anti-programmed deathligand-1 (PD-L1) antibodies. Starting from melanoma, these 
drugs have produced positive results in many solid tumors. Differently from classical chemotherapy and from the majority of molecularly targeted agents that act by directly targeting tumor cells, all the immune checkpoint inhibitors act by targeting the patient's immune system against tumor cells.

First important results have been obtained with ipilimumab in patients affected by malignant melanoma $[5,6]$. Subsequently, also nivolumab and pembrolizumab demonstrated efficacy in these patients [7-9].

Following the results obtained in patients with malignant melanoma, immune checkpoint inhibitors have produced clear evidence of efficacy, within randomized controlled trials, in the treatment of patients with advanced non-smallcell lung cancer (NSCLC). Namely, in patients who have failed first-line platinum-based chemotherapy, nivolumab, pembrolizumab, and atezolizumab, all given as single agents, demonstrated an improvement in overall survival compared to docetaxel [10-13]. In addition, pembrolizumab has also shown superiority compared to platinum-based chemotherapy, when given as first-line in a population of advanced NSCLC patients, selected for the high expression of PD-L1 in tumor cells [14].

Nivolumab has also been approved for the second-line treatment of advanced renal cell cancer, following the results of a randomized phase III trial showing an improvement in overall survival compared to everolimus [15].

Furthermore, the list of other solid tumors where immune checkpoint inhibitors have already produced evidence of activity and efficacy and where these drugs are currently under investigation is long.

\section{Rationale for Immunotherapy in Urothelial Cancer}

The efficacy of immunotherapy in bladder cancer was first established in 1976 when Morales et al. proved for the first time that intravesical instillations of bacillus CalmetteGuérin (BCG) were efficient in preventing recurrences of high-risk nonmuscle invasive urothelial bladder cancer and in treating carcinoma in situ [16]. Although the mechanism of action of BCG is not yet clear even after forty years from the first evidence, it seems to stimulate a cytotoxic response trough the combination of antigenic fragments, processed by bladder cancer cells, with the histocompatibility complex on the tumor cells surface [17].

After this initial success, many other attempts have been made to take advantage of directing T-cells against bladder cancer cells both in the localized and advanced disease, using activating cytokines such as interleukin- (IL-) 2 and interferon- (IFN-) alfa-2B [18, 19]. These drugs have shown limited benefits in achieving disease control.

A turning point took place on the second decade of this century when immune checkpoint inhibitors arrived on the scene. Contrary to the previous strategy this new class of monoclonal antibodies aims to reduce inhibitory signaling instead of directly stimulating T-cells.

The first receptor to be targeted was CTLA-4, a molecule expressed on activated CD4 and CD8 T-cells. CTLA-4 competes with $\mathrm{CD} 28$ for the interaction with the costimulatory
CD80-CD86 molecules on antigen presenting cells (APCs). While the latter interaction promotes T-cells activation and effector functions, CTLA-4-CD80/86 inhibits T-cell activation in lymphoid tissues [20]. Two monoclonal antibodies targeting CTLA-4 have been developed: ipilimumab and tremelimumab, whose effect is to shift T-cell equilibrium toward activation.

It has been further observed that tumor cells might evade immune system surveillance through the interaction between PD-L1 and PD-L2 with their receptor PD-1, which is expressed on CD4 and CD8 T-cells, Tregs, B-cells, and natural killer (NK) cells. Acting directly among tumor microenvironment, drugs directed against either PD-1 or PD-L1 are usually characterized by lower adverse effects than CTLA-4 inhibitors [21].

Furthermore, many other immune checkpoint receptors are currently under investigation in several trials, as potential therapeutic targets. Simultaneously other therapeutic strategies which recruit an adaptive immune response against tumoral antigens might change the natural history of bladder cancer in the near future [20].

Bladder cancer usually shows some biological features that have been associated with better response to immunotherapies. First of all, an adaptive immune response against cancer cells requires the presence tumor antigens endowed with a good immunogenicity. More the mutation board, more likely this kind of antigens is expressed in tumor microenvironment. Bladder cancer is often characterized by a high mutation load. Moreover PD-L1 expression on the surface of tumor cells has been correlated with a higher-stage, suggesting good response to PD1/PD-L1 antagonist, although the results of different trials did not observe the association between PD-L1 expression and tumor response rate [22].

Indeed, the major challenge that is going to be faced in the next years is to find predictive factors granted by greater sensibility and specificity.

\section{PD-L1 Inhibitors}

3.1. Atezolizumab. Atezolizumab is an engineered human monoclonal antibody against PD-L1, able to inhibit the interaction between PD-L1 and its receptor PD-1. A multicentre, nonrandomized, phase II trial (IMVigor 210) evaluated the efficacy and safety profile of intravenous atezolizumab (given every three weeks at the dose of $1200 \mathrm{mg}$ ) in two different cohorts of locally advanced or metastatic urothelial carcinoma: cohort A included treatment naïve patients, ineligible for cisplatin; cohort B included patients progressing during or after platinum-based chemotherapy. PD-L1 expression on tumor infiltrating immune cells was assessed prospectively by immunohistochemistry. On the basis of PD-L1 expression, patients were categorized in three subgroups: IC0 $(<1 \%)$, IC1 $(\geq 1 \%$ but $<5 \%)$, and IC $2 / 3(\geq 5 \%)$.

In cohort $\mathrm{B}$, among the 310 evaluable patients overall response rate (ORR) was $15 \%$ (95\% CI, 11-20) with 5\% of complete responses. High levels of PD-L1 expression were associated with better ORR (27\%; 95\% CI, 19-37). After a median follow-up of 11.7 months, the median duration of response has not yet been reached, and durable responses 
have been recorded also in patients with poor prognostic features [23] (Table 1). Median overall survival was 11.4 months in patients in the IC2/3 group, 8.8 months in the $\mathrm{IC1} / 2 / 3$ group, and 7.9 months in the whole cohort of patients.

Due to these positive results, Food and Drug Administration (FDA) approved in May 2016 atezolizumab for the treatment of patients with locally advanced or metastatic urothelial carcinoma progressing during or following platinumcontaining chemotherapy or within 12 months of either adjuvant or neoadjuvant platinum chemotherapy. The recommended dose is $1200 \mathrm{mg}$, given as an intravenous infusion every three weeks.

As for the cohort A of patients who were not eligible for cisplatin $(n=123)$, the ORR was $23 \%(95 \%$ CI, $16-31)$ in all patients, with slight but not statistically significant differences among PD-L1 subgroups. ORR was 28\% (95\% CI; 14-47) in patients with high PD-L1 expression and 21\% (95\% CI; 9-36) in patients PD-L1 negative. After a 17.2 months median follow-up duration, median overall survival (OS) was 15.9 months (95\% CI; 10.4 to not estimable) in all patients [24] (Table 1).

In the attempt of identifying predictive factors of activity and efficacy of atezolizumab, in addition to PD-L1 determination, authors evaluated also Cancer Genome Atlas gene expression and mutation load. In both cohorts, responses were more frequent in the Luminal II subtype and in patients with higher mutation load, irrespective of PD-L1 expression. Moreover, in cohort B, PD-L1 expression and responses to atezolizumab were most closely associated with immune activation gene expression (e.g., interferon- $\gamma$-inducible Thelper-1-type chemokines: CXCL9 and CXCL10) and CD8 Tcell infiltration $[23,24]$.

Treatment with atezolizumab was well tolerated in both cohorts, with serious adverse events (AEs) occurring in 15$16 \%$ of patients, and only a treatment-related death for sepsis occurred in cohort A $[23,24]$.

A phase 3 trial is evaluating the efficacy of atezolizumab compared to second-line chemotherapy in patients with locally advanced or metastatic urothelial carcinoma progressing to platinum-based treatment; furthermore, several studies are ongoing investigating atezolizumab monotherapy or in combination with chemotherapy or other immunological agents in different stages of disease (Table 2).

3.2. Durvalumab. Durvalumab (MEDI4736) is a selective, high-affinity, human monoclonal antibody directed against PD-L1. A phase I/II multicentre dose escalation and doseexpansion study is ongoing in patients with advanced solid tumors, to evaluate safety, tolerability, and antitumor activity of durvalumab monotherapy. In June 2016, Massard et al. published first results about patients with urothelial carcinoma progressing on or ineligible for cisplatin-based therapy $(n=61)$. Durvalumab, at the dose of $10 \mathrm{mg} / \mathrm{kg}$, was administered intravenously every two weeks, for up to twelve months. Patients were categorized on the basis of PDL1 expression, assessed either on tumor cells or on immune cells (adopting a cutoff of 25\%). Among the 42 patients evaluable for response, the ORR was 31\% (95\% CI, 17.6-47.1). A greater antitumor activity was observed in the PD-L1 positive subgroup (46.4\%; 95\% CI, 27.5-66.1); in the PD-L1 negative patients the ORR was $0 \%(95 \% \mathrm{CI}, 0.0-23.2)$. At the time of analysis, responses were ongoing in 12 of 13 patients with a median duration of response not yet reached (range: 4.1 to 49.3 weeks). Treatment tolerance was optimal; serious AEs occurred in $4.9 \%$ of patients, with no treatment-related deaths [25] (Table 1).

An update of this study has been presented at 2017 ASCO Genitourinary Cancer Symposium. Efficacy analysis included 103 patients with a median follow-up of 7.3 months. The ORR was $20.4 \%$ (13.1-29.5) in the overall population and $29.5 \%$ (18.5-42.6) in the PD-L1 positive subgroup versus $7.7 \%$ (1.6-20.9) in the PD-L1 negative patients [26].

In February 2016 the FDA granted a breakthrough therapy designation to durvalumab as a treatment for PD-L1positive inoperable or metastatic urothelial bladder cancer patients progressing on platinum-based treatment.

Several trials are ongoing in urothelial carcinoma patients investigating activity of durvalumab, alone or in combination with the anti-CTLA4 tremelimumab (Table 2).

3.3. Avelumab. Avelumab is a fully human anti-PD-L1 monoclonal antibody. A large phase Ib trial is ongoing, investigating safety, tolerability, and clinical activity of avelumab in patients with locally advanced or metastatic solid tumors, including patients with urothelial carcinoma whose disease progressed after platinum-based chemotherapy or who were platinum ineligible. Avelumab showed preliminary safety and efficacy in a cohort of 44 patients [27] (Table 1), so an additional cohort of 129 eligible urothelial carcinoma patients was enrolled and received avelumab, $10 \mathrm{mg} / \mathrm{kg}$, every two weeks until confirmed progression, unacceptable toxicity, or withdrawal. Preliminary data about 109 patients with at least four months of follow-up were presented at 2016 ESMO Congress: confirmed ORR was 16.5\% (95\% CI, 10.1-24.8), with 3 complete and 15 partial responses. PFS rate at 12 weeks was 35.6 (95\% CI; 26.5-44.7). Treatment was well tolerated; grade 3-4 treatment-related adverse events occurred in $9 \%$ of patients; and pneumonitis resulted in one treatment-related death [28]. An update of this study was reported at 2017 ASCO Genitourinary Cancer Symposium. Data were available in $153 / 241$ patients with at least six months of followup: ORR was $17.6 \%$ (95\% CI, 12.0-24.6), 88.9\% of responses were ongoing at the time of analysis, and median OS was 7.0 months (95\% CI, 5.6-11.1). Based on a $\geq 5 \%$ PD-L1 expression cutoff assessed prospectively on tumor cells, ORR was significantly higher in PD-L1 positive patients $(25.0 \%$; $95 \% \mathrm{CI}$, 14.4-38.4) compared with PD-L1 negative subgroup (14.7\%; 95\% CI, 7.6-24.7; $p=0.178$ ). Treatment was well tolerated, with only $7.5 \%$ grade $\geq 3$ treatment-related AEs [29].

A randomized, open-label phase 3 trial of avelumab + best supportive care (BSC) versus BSC alone as maintenance therapy after first-line platinum-based chemotherapy is ongoing in patients with advanced urothelial cancer (Table 2).

\section{PD-1 Inhibitors}

4.1. Nivolumab. Nivolumab is a fully human anti-PD-1 monoclonal antibody, currently approved for the treatment for 


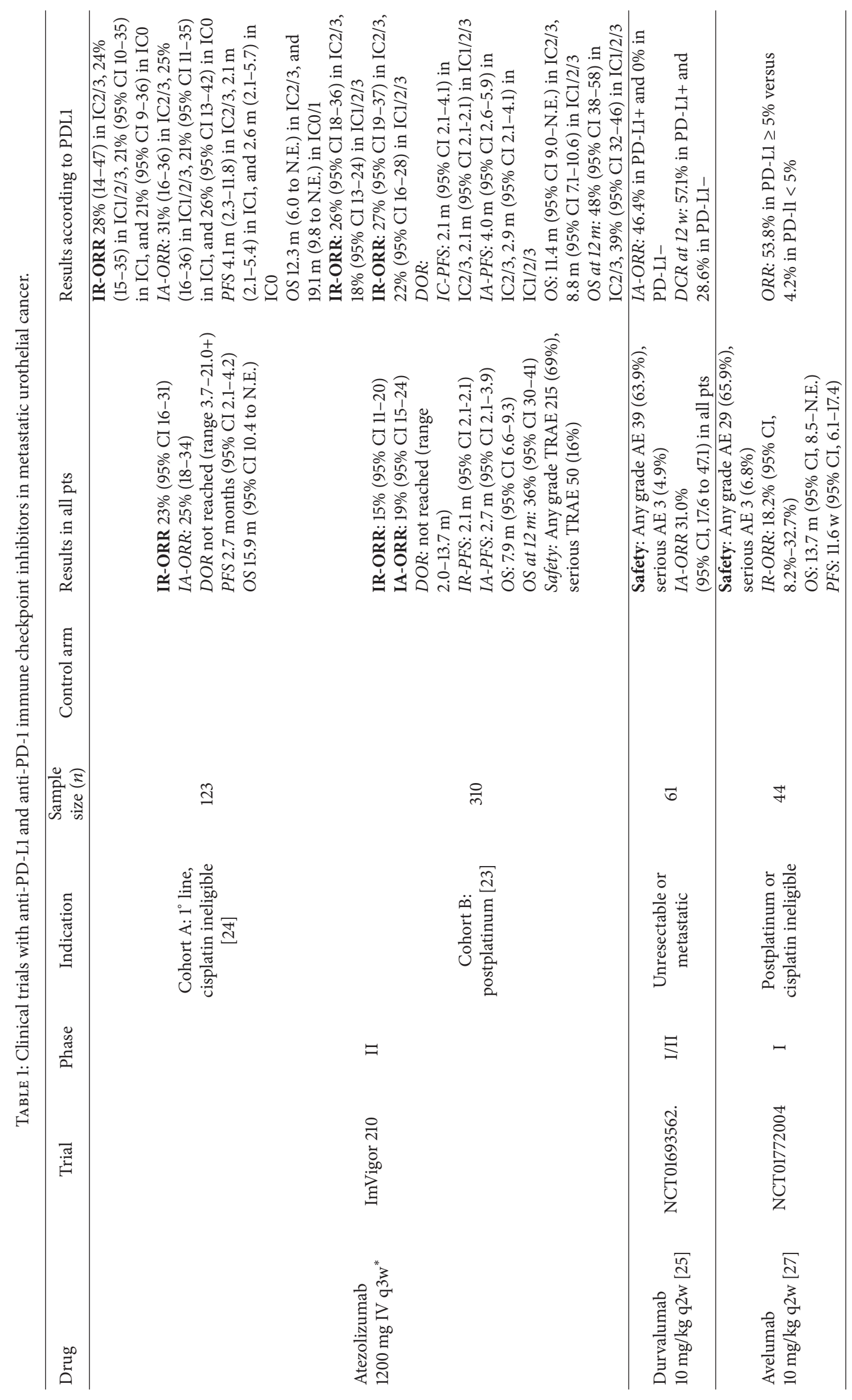




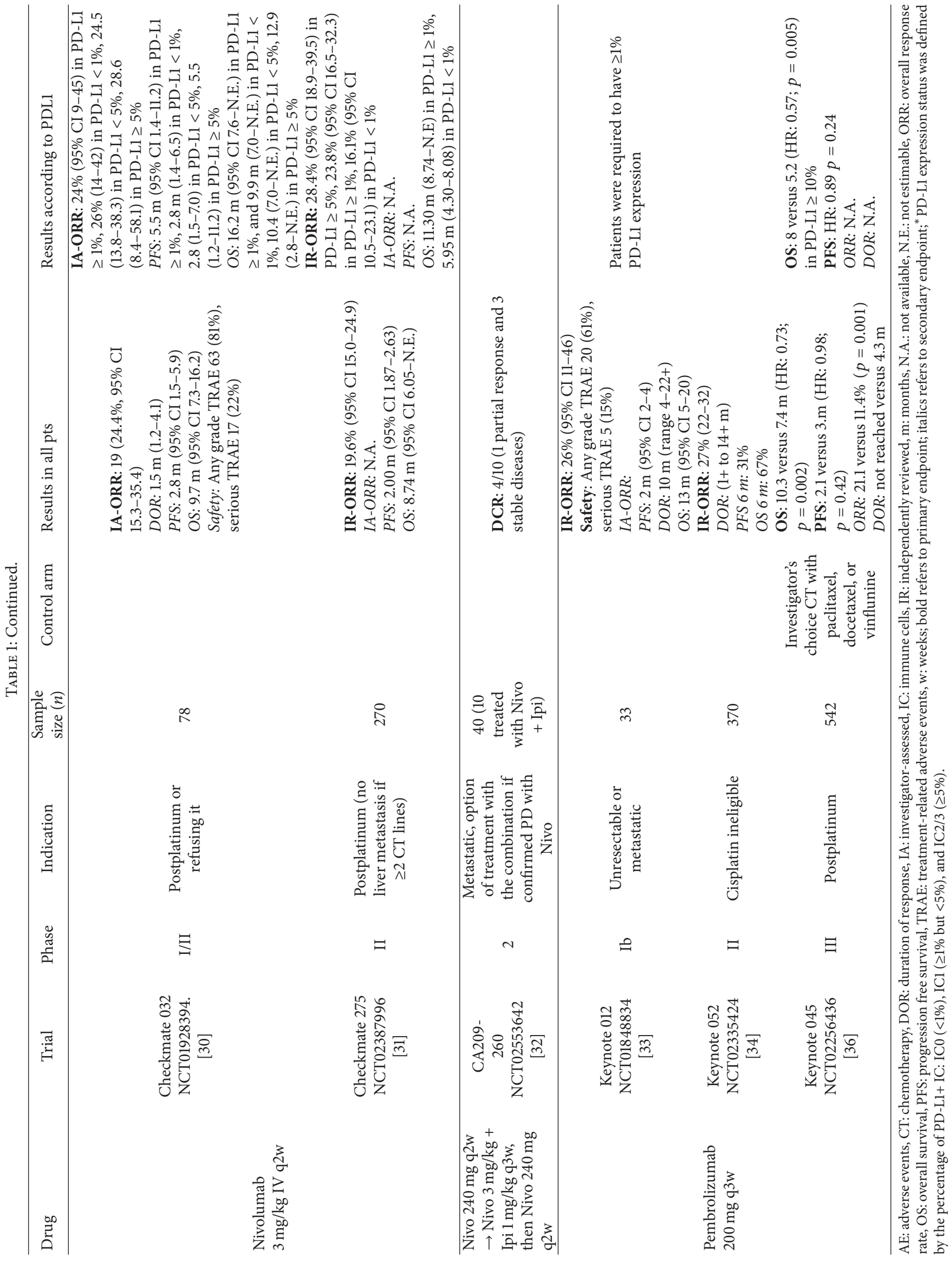


TABLE 2: Ongoing clinical trials of anti PD-L1 and anti PD-1 immune checkpoint inhibitors in metastatic urothelial cancer.

\begin{tabular}{|c|c|c|c|c|c|c|}
\hline & Study & Phase & Regimen & Primary endpoints & $\begin{array}{l}\text { Planned } \\
\text { number of } \\
\text { pts or pts } \\
\text { enrolled }\end{array}$ & Status \\
\hline \multirow[t]{7}{*}{ Atezolizumab } & $\begin{array}{l}\text { NCT02302807 } \\
\text { (IMVigor 211) }\end{array}$ & III & $\begin{array}{c}\text { Atz } 1200 \mathrm{mg} \text { IV dl } \mathrm{q} 3 \mathrm{w} \text { versus } \\
\mathrm{CT}\left(\mathrm{Vnf} 320 \mathrm{mg} / \mathrm{m}^{2} \text { or Txl }\right. \\
\left.175 \mathrm{mg} / \mathrm{m}^{2}, \text { or Txt } 75 \mathrm{mg} / \mathrm{m}^{2}\right) \\
\text { IV d1 q3w }\end{array}$ & OS & 932 & Active, not recruiting \\
\hline & $\begin{array}{l}\text { NCT02807636 } \\
\text { (IMVigor 130) }\end{array}$ & III & $\begin{array}{c}\text { Atz } 1200 \mathrm{mg} \text { IV d1 + CT (Crb } \\
\text { AUC } 4.5 \mathrm{IV} \mathrm{d} 1+\mathrm{Gem} \\
\left.1000 \mathrm{mg} / \mathrm{m}^{2} \mathrm{IV} \mathrm{d} 1,8 \mathrm{q} 3 \mathrm{w}\right) \\
\text { versus Placebo + CT }\end{array}$ & OS, PFS and Safety & 435 & Currently recruiting \\
\hline & NCT02989584 & II & $\begin{array}{l}\text { Atz } 1200 \mathrm{mg} \text { IV d8 q3w + Gem } \\
1000 \mathrm{mg} / \mathrm{m}^{2} \mathrm{IV} \mathrm{d} 1,8+\text { Cis } \\
70 \mathrm{mg} / \mathrm{m}^{2} \mathrm{~d} 1 \mathrm{q} 3 \mathrm{w} \\
\text { (maintenance in phase II) }\end{array}$ & Safety & 30 & Currently recruiting \\
\hline & $\begin{array}{l}\text { NCT02298153 } \\
(\text { ECHO-110) }\end{array}$ & I & $\begin{array}{c}\text { Atz } 1200 \mathrm{mg} \text { IV q3w + } \\
\text { Epacadostat } 25 \text { mg OS BID as } \\
\text { starting dose, followed by dose } \\
\text { escalations. }\end{array}$ & Safety & 118 & Currently recruiting \\
\hline & NCT02928406 & III & Atz 1200 mg IV q3w & Safety & 1000 & Active, not recruiting \\
\hline & NCT02655822 & I & $\begin{array}{c}\text { CPI-444 in } 3 \text { different } \\
\text { schedules versus CPI- } 444+ \\
\text { Atz IV }\end{array}$ & $\begin{array}{l}\text { Safety, ORR, } \\
\text { median AUC of } \\
\text { CPI- } 444\end{array}$ & 534 & Currently recruiting \\
\hline & NCT02543645 & $\mathrm{I} / \mathrm{II}$ & $\begin{array}{l}\text { Varlilumab } 0.3 \text { or } 1 \text { or } 3 \mathrm{mg} / \mathrm{kg} \\
+ \text { Atz } 1200 \mathrm{mg} \text { IV q2w }\end{array}$ & Safety, ORR & 55 & Currently recruiting \\
\hline \multirow[t]{5}{*}{ Durvalumab } & NCT02516241 & III & $\begin{array}{c}\text { IV Drv +/- IV Trm versus CT } \\
\text { (platinum }+ \text { Gem) }\end{array}$ & PFS, OS & 1005 & Active, not recruiting \\
\hline & $\begin{array}{l}\text { NCT02546661 } \\
\text { (Biscay) }\end{array}$ & I & $\begin{array}{c}\text { (A) Drv + AZD4547 } \\
\text { (B) Drv + olaparib } \\
\text { (C) Drv + AZD1775 } \\
\text { (D) Drv } \\
\text { (E) Drv + Vistusertib }\end{array}$ & Safety & 110 & Currently recruiting \\
\hline & NCT02527434 & II & $\begin{array}{l}\text { IV Trm versus IV Trm + IV } \\
\text { Drv versus IV Drv }\end{array}$ & ORR & 66 & Currently recruiting \\
\hline & NCT02643303 & $\mathrm{I} / \mathrm{II}$ & $\begin{array}{c}\text { IV Drv + IV Trm +/- IT/IM } \\
\text { PolyICLC }\end{array}$ & $\begin{array}{l}\text { Recommended } \\
\text { combination dose, } \\
\text { safety, ORR, PFS } \\
\text { and OS }\end{array}$ & 102 & Active, not recruiting \\
\hline & NCT02318277 & $\mathrm{I} / \mathrm{II}$ & $\begin{array}{c}\text { Drv IV q2w + OS } \\
\text { INCB024360 } 25 \text { mg BID } \\
\text { followed by dose escalations. }\end{array}$ & DLT, ORR & 185 & Currently recruiting \\
\hline Avelumab & $\begin{array}{l}\text { NCT02603432 } \\
\text { (JAVELIN } \\
\text { Bladder 100) } \\
\end{array}$ & III & Avl IV q2w + BSC versus BSC & OS & 668 & Currently recruiting \\
\hline \multirow[t]{5}{*}{ Nivolumab } & NCT02387996 & II & IV Niv & ORR & 242 & Active, not recruiting \\
\hline & NCT02897765 & I & $\begin{array}{l}\text { Niv IV } 240 \text { mg q2w +/- } \\
\text { NEO-PV-01 SC + Adj }\end{array}$ & Safety & 90 & Currently recruiting \\
\hline & NCT02496208 & I & $\begin{array}{l}\text { OS cabozantinib-s-malate + } \\
\text { IV Niv +/- IV Ipi }\end{array}$ & Safety and DLT & 66 & Currently recruiting \\
\hline & $\begin{array}{l}\text { NCT01928394 } \\
\text { (Checkmate } \\
\text { 032) }\end{array}$ & $\mathrm{I} / \mathrm{II}$ & $\begin{array}{l}\text { IV Niv +/- IV Ipi (different } \\
\text { schedules) }+/- \text { OS } \\
\text { Cobimetinib }\end{array}$ & ORR & 1150 & Currently recruiting \\
\hline & $\begin{array}{l}\text { NCT02636036 } \\
\text { (SPICE) }\end{array}$ & I & IV Niv + IV Enadenotucirev & MTD & 30 & Currently recruiting \\
\hline
\end{tabular}


TABLE 2: Continued.

\begin{tabular}{|c|c|c|c|c|c|c|}
\hline & Study & Phase & Regimen & Primary endpoints & $\begin{array}{l}\text { Planned } \\
\text { number of } \\
\text { pts or pts } \\
\text { enrolled }\end{array}$ & Status \\
\hline & $\begin{array}{l}\text { NCT02834013 } \\
\text { (DART) }\end{array}$ & II & Niv IV d1,15,29 + Ipi IV d1 q6w & ORR & 334 & Active, not recruiting \\
\hline & NCT02614456 & I & $\begin{array}{c}\text { Phase 1: IFN- } \gamma \text { SC } 50 \mu \mathrm{g} / \mathrm{m}^{2} \\
\mathrm{~d} 1-7 \\
\text { Phase 2: IFN- } \gamma \text { SC QD + Niv } \\
\text { IV d1 q2w } \\
\text { Phase 3: Niv IV d1 q3w }\end{array}$ & Safety, DLT & 15 & Currently recruiting \\
\hline \multirow[t]{21}{*}{ Pembrolizumab } & NCT02717156 & II & $\begin{array}{c}\text { Pmb IV d1 + EphB4-HSA IV } \\
\text { d1,8,15 q3w }\end{array}$ & Safety & 60 & Active, not recruiting \\
\hline & NCT02925533 & I & IV B-701 + IV Pmb q3w & Safety & 12 & Currently recruiting \\
\hline & $\begin{array}{l}\text { NCT02560636 } \\
\text { (PLUMMB) }\end{array}$ & I & IV Pmb + RT & MTD, Safety & 34 & Currently recruiting \\
\hline & $\begin{array}{l}\text { NCT02351739 } \\
\text { (Keynote 143) }\end{array}$ & II & IV Pmb +/- ACP-196 & ORR & 75 & Active, not recruiting \\
\hline & NCT02500121 & II & $\begin{array}{c}\text { Pmb } 200 \text { mg IV d1 q3w versus } \\
\text { placebo }\end{array}$ & 6 months PFS & 200 & Currently recruiting \\
\hline & $\begin{array}{l}\text { NCT02853305 } \\
\text { (Keynote 361) }\end{array}$ & III & $\begin{array}{c}\text { Pmb } 200 \text { mg IV d1 q3w +/- } \\
\text { CT versus CT (platinum + } \\
\text { Gem) }\end{array}$ & PFS, OS & 990 & Currently recruiting \\
\hline & NCT02619253 & I/II & $\begin{array}{l}\text { Pmb } 200 \text { mg IV d1 q3w + } \\
\text { Vorinostat OS d1-14 q3w }\end{array}$ & Safety & 42 & Currently recruiting \\
\hline & NCT02826564 & I & $\begin{array}{l}\text { Stereotactic body } \\
\text { radiotherapy prior to or } \\
\text { concurrent with IV Pmb }\end{array}$ & $\begin{array}{l}\text { Safety, selection of } \\
\text { the sequence arm } \\
\text { with a DLT }<20 \%\end{array}$ & 20 & Currently recruiting \\
\hline & $\begin{array}{l}\text { NCT02880345 } \\
\text { (Radvax) }\end{array}$ & Pilot & $\begin{array}{l}\text { IV Pmb + hypofractionated } \\
\text { RT ( } 2 \text { different regimens) }\end{array}$ & Safety & 14 & Active, not recruiting \\
\hline & NCT02437370 & I & $\begin{array}{c}\text { IV Pmb + IV Txt versus IV } \\
\text { Pmb versus IV Gem }\end{array}$ & MTD & 38 & Currently recruiting \\
\hline & $\begin{array}{l}\text { NCT02043665 } \\
\text { (Keynote 200) }\end{array}$ & I & $\begin{array}{l}\text { (A) CVA21 } \\
\text { (B) } \text { CVA21 + Pmb }\end{array}$ & ORR & 60 & Currently recruiting \\
\hline & NCT02581982 & II & 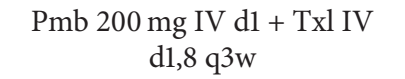 & ORR & 27 & Currently recruiting \\
\hline & NCT01174121 & II & $\begin{array}{c}\text { Cyclophosphamide and } \\
\text { fludarabine }+ \text { Pmb + young } \\
\text { TIL }\end{array}$ & $\begin{array}{l}\text { Rate of tumor } \\
\text { regression }\end{array}$ & 290 & Currently recruiting \\
\hline & NCT03006887 & I & $\begin{array}{c}\text { Pmb } 200 \mathrm{mg} \text { IV d1 + } \\
\text { Lenvatinib OS } 20 \mathrm{mg} \text { QD q3w }\end{array}$ & Safety, DLT & 10 & Active, not recruiting \\
\hline & NCT02501096 & I/II & $\begin{array}{l}\text { Pmb } 200 \mathrm{mg} \text { IV d1 + } \\
\text { Lenvatinib OS QD q3w }\end{array}$ & MTD, ORR, DLT & 250 & Currently recruiting \\
\hline & $\begin{array}{l}\text { NCT02346955 } \\
\text { (MK-6018-001) }\end{array}$ & I & $\begin{array}{l}\text { Multidose escalation of } \\
\text { CM-24 +/- Pmb } 200 \text { mg IV }\end{array}$ & Safety, DLT & 196 & Currently recruiting \\
\hline & NCT02452424 & $\mathrm{I} / \mathrm{II}$ & $\begin{array}{c}\text { Dose escalation of OS } \\
\text { PLX3397 + Pmb } 200 \text { mg IV }\end{array}$ & Safety & 400 & Currently recruiting \\
\hline & NCT02432963 & I & $\begin{array}{c}\text { IV Pmb + SC MVA-p53 } \\
\text { Vaccine }\end{array}$ & Tolerability & 19 & Currently recruiting \\
\hline & NCT02393248 & I/II & $\begin{array}{c}\text { Phase 1: dose } \\
\text { escalation/expansion of } \\
\text { INCB054828 } \\
\text { Phase 2: INCB054828 + } \\
\text { Pmb/CT (Txt or Cis + Gem) }\end{array}$ & $\begin{array}{c}\text { MTD, } \\
\text { pharmacodynamics }\end{array}$ & 150 & Currently recruiting \\
\hline & NCT02443324 & I & IV Pmb + Ramucirumab IV & DLT & 155 & Currently recruiting \\
\hline & NCT02856425 & I & IV Pmb + OS Nintedanib & MTD & 18 & Currently recruiting \\
\hline
\end{tabular}

Atz: atezolizumab; Avl: avelumab; Cis: cisplatin; Drv: durvalumab; Gem: gemcitabine Ipi: ipilimumab; Trm: tremelimumab; Txl: taxol; Txt: taxotere; Niv: nivolumab; Pmb: pembrolizumab. 
TABLE 3: Ongoing clinical trials of anti-CTLA-4 immune checkpoint inhibitors in metastatic urothelial cancer.

\begin{tabular}{|c|c|c|c|c|c|c|}
\hline & Study & Phase & Regimen & $\begin{array}{l}\text { Primary } \\
\text { endpoints }\end{array}$ & $\begin{array}{l}\text { Planned } \\
\text { number of } \\
\text { pts or pts } \\
\text { enrolled }\end{array}$ & Status \\
\hline \multirow{5}{*}{ Ipilimumab } & NCT01524991 & II & $\begin{array}{l}\text { IV gemcitabine } 1000 \mathrm{mg} / \mathrm{m}^{2} \mathrm{~d} \\
1,8+\text { cisplatin } 70 \mathrm{mg} / \mathrm{m}^{2} \mathrm{~d} 1 \mathrm{q} 3 \mathrm{w} \\
\text { IV Ipi } 10 \mathrm{mg} / \mathrm{kg} \mathrm{d} 1 \text { (start c3) }\end{array}$ & 1 year OS & 36 & Active, not recruiting \\
\hline & NCT02496208 & I & $\begin{array}{l}\text { OS cabozantinib-s-malate + IV } \\
\text { Niv +/- Ipi }\end{array}$ & Safety and DLT & 66 & Currently recruiting \\
\hline & NCT01928394 & I/II & $\begin{array}{c}\text { IV Niv +/- Ipi (different } \\
\text { schedules) +/- cobimetinib }\end{array}$ & ORR & 1150 & Currently recruiting \\
\hline & NCT02381314 & I & $\begin{array}{c}\text { IV Ipi d1 q3w + IV } \\
\text { enoblituzumab weekly }\end{array}$ & Safety & 84 & Currently recruiting \\
\hline & NCT02834013 (DART) & II & $\begin{array}{c}\text { IV Niv d 1,15,29 + IV ipilimumab } \\
\text { d1 q6w }\end{array}$ & ORR & 334 & Active, not recruiting \\
\hline \multirow{3}{*}{ Tremelimumab } & NCT02516241 & III & $\begin{array}{l}\text { IV Drv +/- IV Trm versus CT } \\
\text { (platinum + gemcitabine) }\end{array}$ & PFS, OS & 1005 & Active, not recruiting \\
\hline & NCT02527434 & II & $\begin{array}{c}\text { IV Trm versus IV Trm + IV Drv } \\
\text { versus IV Drv }\end{array}$ & ORR & 66 & Currently recruiting \\
\hline & NCT02643303 & $\mathrm{I} / \mathrm{II}$ & $\begin{array}{l}\text { IV Drv + IV tremelimumab +/- } \\
\text { IT/IM PolyICLC }\end{array}$ & $\begin{array}{l}\text { Recommended } \\
\text { combination } \\
\text { dose, safety, ORR, } \\
\text { PFS, and OS }\end{array}$ & 102 & Active, not recruiting \\
\hline
\end{tabular}

different malignancies, as front-line (melanoma) or secondline monotherapy (NSCLC, renal cell cancer) or in combination with ipilimumab (melanoma). An ongoing openlabel, two-stage, multiarm, phase I/II trial, Checkmate 032, is evaluating safety and activity of nivolumab alone or in combination with ipilimumab in subjects with advanced or metastatic solid tumors. First results about a cohort of patients with advanced urothelial carcinoma, who progressed during or after platinum-based chemotherapy, treated with nivolumab alone ( $3 \mathrm{mg} / \mathrm{kg}$ intravenously every two weeks), were published in October 2016. Eligible patients were enrolled regardless of tumor cells PD-L1 expression that was assessed retrospectively in pretreatment tumor biopsy specimens collected within three months before treatment start. A confirmed ORR was achieved in $24.4 \%$ (95\% CI, 15.3-35.4) of 78 patients treated with nivolumab monotherapy, regardless of PD-L1 tumor expression. There was no difference in the ORR between patients with PD-L1 expression lower than $1 \%$ $(26.2 \%)$ and patients with PD-L1 expression $\geq 1 \%(24.0 \%)$. However median OS was over 16.2 months in PD-L1 positive tumors and 9.9 months in PD-L1 negative ones [30] (Table 1).

These data were recently confirmed by positive results of phase II study, Checkmate 275, evaluating activity and safety of nivolumab in 270 patients with metastatic bladder cancer progressing during or after first-line platinumbased chemotherapy. Confirmed ORR was 19.6\% (95\% CI, 15.0-24.9) for all patients, $28.4 \%$ for patients with PD-L1 expression of $5 \%$ or greater, $23.8 \%$ for patients with PD-L1 expression of $1 \%$ or greater, and $16.1 \%$ for patients with PD-L1 expression of less than $1 \%$. After a median follow-up equal to 7 months, $24.4 \%$ of patients were still on treatment. Median OS was 8.74 months in the whole study population; in the subgroup of patients expressing PD-L1 $\geq 1 \%$ on tumor cells median OS was 11.3 months, while in PD-L1 negative patients it was 5.95 months. Cancer Genome Atlas gene expression was also analysed on pretreatment tumor tissue: responses were more frequent in the Basal I subtype according to Atlas classification, which showed the strongest association with interferon-y signature and the highest CD8 expression [31] (Table 1).

At 2017 ASCO Genitourinary Cancer Symposium, preliminary data about combination of nivolumab and ipilimumab have been presented. Ten patients with advanced or metastatic urothelial cancer, refractory to nivolumab monotherapy, were treated. Despite a slight increase of immune-related toxicities, treatment was well tolerated and showed a promising activity, with a disease control rate of $40 \%$ (one partial response and three stable disease were reported) [32]. Of course, the number of patients described in this preliminary experience is still too small to comment the activity of the combination. Trials ongoing evaluating nivolumab in combination with ipilimumab are shown in Table 3 and will clarify the real potential of the immunetherapy combination.

4.2. Pembrolizumab. Pembrolizumab is a humanized monoclonal antibody directed against PD-1, which has shown promising results for treatment of metastatic bladder cancer. Results about urothelial cancer patients' cohort of the nonrandomized, multicohort, open-label, phase $1 \mathrm{~b}$ Keynote 012 basket trial were published in January 2017. Thirtythree patients with advanced or metastatic urothelial cancer with at least $1 \% \mathrm{PD}-\mathrm{L} 1$ expression in tumor cells or stroma 
were enrolled and treated with $10 \mathrm{mg} / \mathrm{kg}$ intravenous pembrolizumab every two weeks, until progressive disease or unacceptable toxicity. Treatment was generally well tolerated, and only $9 \%$ of patients experienced serious adverse events. Seven of 27 evaluable patients (26.0\%; 95\% CI, 11.0-46.0) achieved partial or completed responses [33] (Table 1).

At 2017 ASCO Genitourinary Cancer Symposium, preliminary data of phase II Keynote 052 trial have been presented. In detail, this trial evaluated activity and safety of pembrolizumab in cisplatin-ineligible patients with metastatic or locally advanced bladder cancer, enrolled regardless of PD-L1 expression. However, PD-L1 expression was prospectively assessed in tumor and immune cells, to better characterize responding and nonresponding patients. Patients received pembrolizumab $200 \mathrm{mg}$ intravenously every three weeks, for up to 24 months of treatment. Among patients with at least four months of follow-up, ORR was $27 \%$ (95\% CI, 22-32); no data about activity according to PD-L1 expression were reported. The only data available have been presented at ESMO 2016 Congress: ORR was 36.7\% (95\% CI, 19.9-56.1) in patients with $10 \%$ or greater PD-L1 expression [34, 35] (Table 1).

Of note, a randomized phase III trial, Keynote 045 study, compared pembrolizumab to chemotherapy (consisting of either paclitaxel, docetaxel, or vinflunine according to Investigator's choice) in 542 patients with locally advanced unresectable or metastatic urothelial carcinoma recurring or progressing following platinum-based chemotherapy. A survival benefit was shown in the pembrolizumab group (median OS was 10.3 versus 7.4 months, hazard ratio for death, $0.73 ; 95 \%$ CI, 0.59 to $0.91 ; p=0.002$ ), regardless of PD-L1 expression; also ORR was significantly improved in the pembrolizumab group (21.1\% versus $11.4 \% ; p=0.001)$. These benefits were similar across almost all subgroups examined, regardless of the type of chemotherapy or the presence of poor prognostic factors, such as hepatic metastases. Fewer adverse events for any grade occurred in the pembrolizumab group compared to patients treated with chemotherapy (Table 1) [36].

Various studies are ongoing investigating pembrolizumab activity in combination with other systemic therapies and radiotherapy (Table 2 ).

\section{Drugs That Target CTLA-4}

5.1. Ipilimumab and Tremelimumab. Safety and immunologic pharmacodynamic effects of ipilimumab, an anti-CTLA-4 monoclonal antibody, have already been evaluated in the neoadjuvant setting in a small phase II clinical trial. Twelve patients with localized, high grade, urothelial carcinoma of the bladder were treated with ipilimumab, at the dose of 3 or $10 \mathrm{mg} / \mathrm{kg}$. Safety profile of treatment was good. In all patients, an increase in CD $4+\mathrm{T}$-cell population in both tumor tissue and peripheral blood was found, probably positively related to clinical benefit. Of note, eight patients showed tumor regression: on radical cystectomy specimens, obtained after neoadjuvant treatment, lower stages of disease were found [37].
Several trials are now ongoing, to evaluate anti CTLA-4 antibody ipilimumab or tremelimumab, alone or in combination with nivolumab or durvalumab or chemotherapy or other target therapies (Table 3 ). Results are not yet available.

\section{Other Immunotherapies}

Immunotherapy includes treatments that work in different ways, not only limited to anti-PD-1, anti-PD-L1, or anti-CTL A4 antibodies. There are many potential targets under study: antigens on tumor cells surface, new immune-checkpoints, and tumor microenvironment. Against some of these targets, vaccines and monoclonal antibody are on development, even if few results from clinical trials are available at the time.

\subsection{Immune System Targets}

6.1.1. Recombinant Interleukin-2. One of the first attempts of immunotherapy foresaw the use of recombinant interleukin2 (rIL-2), a cytokine whose main function is to promote Tcell differentiation and activation. In 1991, nine patients with metastatic transitional bladder cancer were treated with a continuous infusion of rIL-2 associated with lymphocytes previously stimulated in vitro with the same cytokine. Unfortunately none of the patients benefited from that treatment: at the first radiological evaluation eight patients showed progression disease and one patient had stable disease [18].

6.1.2. ALT-801. More recently at ASCO 2015 annual meeting, preliminary results of a phase Ib/II study of cisplatin and gemcitabine in combination with ALT-801, an IL-2/T-cell receptor fusion protein, in advanced or metastatic urothelial carcinoma were presented. Dose escalation expansion cohort phase Ib study included both chemonaïve and chemorefractory patients (group 1), whereas phase II expansion study included only chemorefractory patients (group 2). 34 of the 62 enrolled patients were chemorefractory. Among these patients, ORR was 35\% (95\% CI: 20-54\%), and median OS was 12.3 months for group 1 (data not available for group 2 and for chemonaïve patients). Almost all patients experienced severe hematological toxicities [38].

6.1.3. B7-H3. B7H3, also known as $\mathrm{CD} 276$, is a ligand of the $\mathrm{B} 7$ family, which also includes the better known PD-1 and PD-L1. Even if its receptor remains unidentified, B7H3 acts as coinhibitor of peripheral immune response, and its expression seems to be particularly intense in urothelial carcinoma and could correlate with poor prognosis [39]. A dose escalation phase I trial is ongoing (NCT01391143) to evaluate toxicity and potential antitumor activity of the monoclonal antibody MGA271 (enoblituzumab), in patients with various refractory cancers, including urothelial cancer that express B7H3 antigen. Preliminary data were presented at the 2015 Society for Immunotherapy of Cancer (SITC) Annual Meeting. Treatment showed an optimal tolerability with few severe adverse events and a promising activity in patients with melanoma, prostate, and bladder cancer [40].

Another phase I trial (NCT02628535) is currently recruiting participants to assess safety and establish the maximum 
tolerated dose (MTD) of MGD009, a humanized, DualAffinity Retargeting, or DART ${ }^{\circledR}$ molecule that recognizes both B7-H3 and CD3. Patients must have B7-H3 positive unresectable locally advanced or metastatic tumors, including bladder cancer.

6.1.4. $O X-40$ and 4-1bb. OX-40 and 4-1bb, also known respectively as CD134 and CD137, are both members of the Tumor Necrosis Factor receptor (TNF-r) super-family. The former is expressed on CD4 and CD8 T-cell surfaces, the later on NK and activated T-cells. The activation of both signal pathways promotes T-cell proliferation and survival. Moreover OX-40 provides a stimulatory signal to effectors and memory T-cell population, and an inhibitory signal to regulatory T-cells $[41,42]$.

A phase I dose escalation study (NCT02315066) is currently recruiting participants to assess safety and potential activity of an experimental OX-40 agonist alone or in combination with a $4-1 \mathrm{bb}$ agonist, in patients with various tumors, including urothelial bladder carcinoma.

6.1.5. TILs. Another promising therapeutic strategy is the infusion of externally manipulated T-cells that could be extracted from tumor tissue, the so-called tumor infiltrating lymphocytes or TILs, to give rapid immunity. TILs could be simple expanded ex vivo or selected according to recognized antigens. In a small open trial twelve patients underwent surgery for stage IV bladder cancer and TILs from lymph nodes draining metastatic tumors were collected. In six of them, lymphocytes were infused after in vitro expansion without any severe AEs. No data are available on the efficacy of the treatment [43]. A phase II trial (NCT01174121) is now recruiting patients with metastatic solid tumors, including urothelial bladder cancer, with at least one resectable lesion for TILs generation. Lymphocytes will be reinfused after conditioning chemotherapy and pembrolizumab administration. Results of another trial (NCT02863913), not yet open for participants' recruitment, will add important evidence. It is a phase I dose escalation clinical trial to assess the safety of PD1 knockout engineered T-cells in treating metastatic advanced bladder cancer.

\subsection{Tumor Targets}

6.2.1. HER2. Human epidermal growth factor receptor 2 (HER2), also known as CD340, is a member of a big receptor family, encoded by a protooncogene whose alterations (almost amplification and overexpression) are common not only in breast and gastric, but also in urothelial cancer. HER2 target therapy had shown interesting activity in preclinical studies and phase I clinical trials. Unfortunately no difference in efficacy on addiction of trastuzumab to standard chemotherapy with platinum and gemcitabine was detected in advanced or metastatic urothelial carcinoma overexpressing HER2 in a phase II clinical trial [44]. At 2017 ASCO Genitourinary Cancer Symposium preliminary results of the ongoing phase IIA MyPathway trial were presented. Twelve patients with platinum-resistant HER2-positive metastatic urothelial cancer have been enrolled, and at a median followup of 5.4 months there were a CR, two PR, and two stabilisation of disease [45]. Other clinical trials are still ongoing in these patients, testing other HER2 inhibitors, like trastuzumab emtansine (NCT02999672) and Lapatinib (NCT00949455, NCT02342587).

Alternative strategies are under development, looking at HER-2 as a target for immunotherapy. A dendritic cell vaccine called AdHER2, created using an individual's own immune cells, has been developed to stimulate the immune system to recognize HER-2. A phase I study (NCT01730118) is now recruiting patients with various solid tumors and HER2 overexpression [46].

6.2.2. hCG- $\beta$. Human Chorionic Gonadotropin beta-chain (hCG- $\beta$ ) is an antigen frequently expressed by epithelial malignancies, including urothelial cancer. Elevated hCG- $\beta$ serum levels and/or tissue expression are associated with a more aggressive disease course. CDX-1307 is a human monoclonal antibody against the APC mannose receptor fused to hCG- $\beta$ that acts like a vaccine. Internalized by APCs, CDX-1307 is processed and hCG- $\beta$ is presented as an antigen, inducing specific cellular and humoral immune response.

A phase I trial demonstrated that CDX-1307 is well tolerated and active, inducing consistent humoral and T-cell responses when coadministrated with Granulocyte-Macrophage Colony-Stimulating Factor (GM-CSF) and Tall Like Receptor (TLR) agonists, in patients with advanced epithelial malignancies, including bladder cancer [47]. A phase II trial (NCT01094496) to evaluate antitumor activity before and after bladder surgery has recently been completed, but results are not available.

6.2.3. MAGE-A. Melanoma associated antigen A (MAGE-A) are a family of tumor specific antigens expressed in several cancer cell types, but not in normal tissue, except for the testis. MAGE-A proteins are recognized by cytotoxic T-cells and are promising targets for immunotherapy [48]. Partial or complete responses after MAGE-A target immunotherapy have been reported, also for advanced bladder cancer. Particularly three of four heavily pretreated patients with high expression of MAGE-A were enrolled in a small pilot clinical trial. They were treated with subcutaneous injections of autologous dendritic cells pulsed with MAGE-A3 epitopes peptides, showing significant reduction of tumor burden [49].

In a phase I/early II trial, patients with stage III or IV malignancies, including three with bladder cancer, all MAGE-3 positive, were randomized to three different escalation dose levels of a recombinant MAGE-3 vaccine, associated with fixed doses of an immunological adjuvant, in order to further improve its immunogenicity. One of the bladder cancer patients showed a short-term almost complete response of two months [50].

A dose escalation phase I trial (NCT02989064) is now recruiting patients with MAGE-410 positive advanced malignancies, including bladder cancer. Treatment protocol provides the administration of autologous genetically modified 
MAGE A10 T-cells, and the primary endpoint is the evaluation of safety and tolerability of that treatment.

6.2.4. NY-ESO-1. NY-ESO-1 is one of the most immunogenic tumor antigens, expressed in cancer and testis, but not in other normal tissues (similarly to MAGE-A1). It is expressed in approximately $25-30 \%$ of bladder cancers, highly in advanced stages, and it is considered one of the best targets for T-cell receptor (TCR) based immunotherapy in solid cancers. NY-ESO-1-specific T-cell responses, induced in cancer patients using NY-ESO-1 peptides, proteins, and viruses encoding NY-ESO-1, are too weak to eradicate tumor cells [51]. So to improve clinical response TCR gene therapy is being developed. Two phase I trials (NCT02457650 and NCT02869217) are currently recruiting participants with NYESO-1-expressing malignancies to evaluate the safety and feasibility of the administration of anti-NY-ESO-1 TCR engineered autologous T-cells.

6.3. Peptide Personalized Vaccination. In the context of an increasingly personalized medicine, an open-label, randomized phase II trial evaluated safety and efficacy of peptide personalized vaccination compared to best supportive care in eighty patients with advanced urothelial bladder cancer progressing after platinum-containing chemotherapy. Vaccination consisted in subcutaneous injections of maximum four peptides chosen from a pool of thirty-one peptides according to patients HLA type and specific peptide-reactive IgG titers. PR was observed in 9 (23\%) patients in the experimental arm, with mo CR. A significant improvement in OS was also noted (HR, 0.58; 95\% CI, 0.34-0.99, $p=0.049$ ), but not in PFS. Treatment was fairly well tolerated; almost all AEs were of grade 1 or 2; there were no grade 4 AEs or treatment-related deaths. Obviously, as the authors concluded, further largescale, randomized trials are needed to confirm these results [52].

\section{Discussion}

The encouraging results recently obtained with several immune checkpoint inhibitors [23-25, 27, 31, 36] raise enthusiasm about the future role of this therapeutic approach for patients affected by advanced urothelial cancer. As well known, standard treatment for these patients is platinumbased chemotherapy, characterized by a difficult balance between efficacy and treatment toxicity. The availability of immune checkpoint inhibitors, both in patients who are considered medically unfit for cisplatin and in patients who have experienced disease progression after chemotherapy, represents a clinically valuable opportunity. Interestingly, a nonnegligible proportion of patients experience a durable disease control, with a chance of long-survival that has been observed, with the use of these drugs, in many types of solid tumors.

However, similar to what is occurring also in other tumors, knowledge about predictive factors of efficacy and patients' selection criteria for immunotherapy is still not ideal and, within all the clinical trials, a relevant number of patients failed to respond to the PD-1/PD-L1 checkpoint blockades. From this point of view, it would be crucial to identify a biomarker to predict the response to checkpoint blockades. In principle, a perfect positive predictive value could allow avoiding treating patients who are not going to obtain any benefit, while a perfect negative predictive value could allow not denying treatment to any of the patients who could potentially benefit. Unfortunately, at the moment, we have no biomarker with a good positive and negative predictive value. The expression of PD-L1 has been studied as a putative biomarker in many of the trials testing antiPD-1 and anti-PD-L1 drugs, but PD-L1 staining cannot be used to accurately select patients for PD-1/PD-L1 pathway blockade due to the low prediction accuracy and dynamic changes [53]. Interestingly, tumor infiltrating immune cells and molecules in the tumor microenvironment, alone or along with PD-L1 expression, could be useful as predictive factors [53]. Furthermore, gene analysis (gene signatures, mutational landscape, and/or mismatch-repair deficiency) could be useful if interesting preliminary evidence will be confirmed and validated in further studies [23, 24].

The diffusion of immune checkpoint inhibitors in clinical practice will imply the confidence of medical oncologists with the diagnosis and management of typical side effects associated with this therapeutic approach [54].

As for the applicability of trials results in clinical practice, reasonably, we will have no direct comparisons between different anti-PD-1 and anti-PD-L1 that are currently undergoing the clinical development. In the absence of obvious differences in efficacy emerged in indirect comparison of clinical trials, we do not know which is the best treatment choice. Other issues that are not completely answered by the evidence produced in clinical trials are the dose-response relationship (recent evidence in melanoma with ipilimumab suggests that higher dose is associated with higher efficacy [55]) and the optimal duration of treatment (continuous until disease progression or with planned "stop-and-go").

Currently ongoing trials will clarify the role of immune checkpoint agents as first-line treatment, compared to platinum-based chemotherapy. Based on the results of these trials, along with the trials testing other categories of immune treatments, treatment paradigm for patients affected by advanced urothelial cancer could be soon radically changed compared to current guidelines.

\section{Conflicts of Interest}

The authors declare that there are no conflicts of interest regarding the publication of this paper.

\section{Authors' Contributions}

Clizia Zichi and Marcello Tucci equally contributed to the review.

\section{References}

[1] C. G. Drake, E. Jaffee, and D. M. Pardoll, "Mechanisms of immune evasion by tumors," Advances in Immunology, vol. 90, pp. 51-81, 2006. 
[2] M. D. Vesely, M. H. Kershaw, R. D. Schreiber, and M. J. Smyth, "Natural innate and adaptive immunity to cancer," Annual Review of Immunology, vol. 29, pp. 235-271, 2011.

[3] I. Mellman, G. Coukos, and G. Dranoff, "Cancer immunotherapy comes of age," Nature, vol. 480, no. 7378, pp. 480-489, 2011.

[4] S. P. Kerkar and N. P. Restifo, "Cellular constituents of immune escape within the tumor microenvironment," Cancer Research, vol. 72, no. 13, pp. 3125-3130, 2012.

[5] F. S. Hodi, S. J. O’Day, D. F. McDermott et al., "Improved survival with ipilimumab in patients with metastatic melanoma," The New England Journal of Medicine, vol. 363, no. 8, pp. 711723, 2010.

[6] C. Robert, L. Thomas, and I. Bondarenko, "Ipilimumab plus dacarbazine for previously untreated metastatic melanoma," The New England Journal of Medicine, vol. 364, no. 26, pp. 25172526, 2011.

[7] J. Larkin, V. Chiarion-Sileni, R. Gonzalez et al., "Combined nivolumab and ipilimumab or monotherapy in untreated melanoma," New England Journal of Medicine, vol. 373, no. 1, pp. 23-34, 2015.

[8] C. Robert, J. Schachter, G. V. Long et al., "Pembrolizumab versus ipilimumab in advanced melanoma," The New England Journal of Medicine, vol. 372, no. 26, pp. 2521-2532, 2015.

[9] F. S. Hodi, J. Chesney, A. C. Pavlick et al., "Combined nivolumab and ipilimumab versus ipilimumab alone in patients with advanced melanoma: 2-year overall survival outcomes in a multicentre, randomised, controlled, phase 2 trial," The Lancet Oncology, vol. 17, no. 11, pp. 1558-1568, 2016.

[10] J. Brahmer, K. L. Reckamp, P. Baas et al., "Nivolumab versus docetaxel in advanced squamous-cell non-small-cell lung cancer," The New England Journal of Medicine, vol. 373, no. 2, pp. 123-135, 2015.

[11] R. S. Herbst, P. Baas, D.-W. Kim et al., "Pembrolizumab versus docetaxel for previously treated, PD-L1-positive, advanced nonsmall-cell lung cancer (KEYNOTE-010): a randomised controlled trial," The Lancet, vol. 387, no. 10027, pp. 1540-1550, 2016.

[12] H. Borghaei, L. Paz-Ares, L. Horn et al., "Nivolumab versus docetaxel in advanced nonsquamous non-small-cell lung cancer," The New England Journal of Medicine, vol. 373, no. 17, pp. 16271639, 2015.

[13] A. Rittmeyer, F. Barlesi, D. Waterkamp et al., "Atezolizumab versus docetaxel in patients with previously treated non-small-cell lung cancer $(\mathrm{OAK})$ : a phase 3, open-label, multicentre randomised controlled trial," The Lancet, vol. 389, no. 10066, pp. 255-265, 2017.

[14] M. Reck, D. Rodríguez-Abreu, A. G. Robinson et al., "Pembrolizumab versus chemotherapy for PD-L1-positive non-smallcell lung cancer," New England Journal of Medicine, vol. 375, no. 19, pp. 1823-1833, 2016.

[15] R. J. Motzer, B. Escudier, D. F. McDermott et al., "Nivolumab versus everolimus in advanced renal-cell carcinoma," New England Journal of Medicine, vol. 373, no. 19, pp. 1803-1813, 2015.

[16] A. Morales, D. Eidinger, and A. W. Bruce, "Intracavitary bacillus calmette guerin in the treatment of superficial bladder tumors," Journal of Urology, vol. 116, no. 2, pp. 180-183, 1976.

[17] J. Bellmunt, T. Powles, and N. J. Vogelzang, "A review on the evolution of PD-1/PD-L1 immunotherapy for bladder cancer: the future is now," Cancer Treatment Reviews, vol. 54, pp. 58-67, 2017.

[18] G. Gautier Hermann, P. Flemming Geertsen, H. von der Maase et al., "Recombinant interleukin-2 and lymphokine-activated killer cell treatment of advanced bladder cancer: clinical results and immunological effects," Cancer Research, vol. 52, no. 3, pp. 726-733, 1992.

[19] F. N. Joudi, B. J. Smith, and M. A. O’Donnell, “Final results from a national multicenter phase II trial of combination bacillus Calmette-Guérin plus interferon $\alpha$-2B for reducing recurrence of superficial bladder cancer," Urologic Oncology: Seminars and Original Investigations, vol. 24, no. 4, pp. 344-348, 2006.

[20] N. M. Donin, A. T. Lenis, S. Holden et al., "Immunotherapy for the treatment of urothelial carcinoma," The Journal of Urology, vol. 197, no. 1, pp. 14-22, 2017.

[21] F. Fakhrejahani, Y. Tomita, A. Maj-Hes, J. B. Trepel, M. de Santis, and A. B. Apolo, "Immunotherapies for bladder cancer: a new hope," Current Opinion in Urology, vol. 25, no. 6, pp. 586-596, 2015.

[22] S. A. Mullane and J. Bellmunt, "Cancer immunotherapy," Current Opinion in Urology, vol. 26, no. 6, pp. 556-563, 2016.

[23] J. E. Rosenberg, J. H. Censits, T. Powles et al., "Atezolizumab in patients with locally advanced and metastatic urothelial carcinoma who have progressed following treatment with platinumbased chemotherapy: a single-arm, multicentre, phase 2 trial," Lancet, vol. 387, pp. 1909-1920, 2016.

[24] A. V. Balar, M. D. Galsky, J. E. Rosenberg et al., "Atezolizumab as first-line treatment in cisplatin-ineligible patients with locally advanced and metastatic urothelial carcinoma: a single-arm, multicentre, phase 2 trial," The Lancet, vol. 389, no. 10064, pp. 67-76, 2017.

[25] C. Massard, M. S. Gordon, S. Sharma et al., "Safety and efficacy of durvalumab (MEDI4736), an anti-programmed cell death ligand-1 immune checkpoint inhibitor, in patients with advanced urothelial bladder cancer," Journal of Clinical Oncology, vol. 34, no. 26, pp. 3119-3125, 2016.

[26] T. Powles, P. H. O’Donnell, C. Massard et al., "Updated efficacy and tolerability of durvalumab in locally advanced or metastatic urothelial carcinoma," Journal of Clinical Oncology, vol. 35, supplement 6S, 2017, abstract 286.

[27] A. B. Apolo, J. R. Infante, A. Balmanoukian et al., "Avelumab, an anti-programmed death-ligand 1 antibody, in patients with refractory metastatic urothelial carcinoma: results from a multicenter, phase ib study," Journal of Clinical Oncology, 2017.

[28] M. Patel, J. Ellerton, M. Agrawal et al., "Avelumab (MSB0010718C; anti-PD-L1) in patients with metastatic urothelial carcinoma progressed after platinum-based therapy or platinum ineligible," Annals of Oncology, vol. 27, no. 6, pp. 266-295, 2016.

[29] M. R. Patel, J. A. Ellerton, J. R. Infante et al., "Avelumab in patients with metastatic urothelial carcinoma: pooled results from two cohorts of the phase lb JAVELIN solid tumor trial," Journal of Clinical Oncology, vol. 35, supplement 6S, 2017, abstract 330 .

[30] P. Sharma, M. K. Callahan, P. Bono et al., "Nivolumab monotherapy in recurrent metastatic urothelial carcinoma (CheckMate 032): a multicentre, open-label, two-stage, multi-arm, phase 1/2 trial," The Lancet Oncology, vol. 17, no. 11, pp. 1590$1598,2016$.

[31] P. Sharma, M. Retz, A. Siefker-Radtke et al., "Nivolumab in metastatic urothelial carcinoma after platinum therapy (CheckMate 275): a multicentre, single-arm, phase 2 trial," The Lancet Oncology, vol. 18, no. 3, pp. 312-322, 2017.

[32] M. K. Callahan, B. E. Kania, G. Iyer et al., "Evaluation of the clinical activity of ipilimumab (IPI) plus nivolumab (NIVO) in 
patients (pts) with NIVO-refractory metastatic urothelial cancer (UC)," Journal of Clinical Oncology, vol. 35, supplement 6S, 2017, abstract 384 .

[33] E. R. Plimack, J. Bellmunt, S. Gupta et al., "Safety and activity of pembrolizumab in patients with locally advanced or metastatic urothelial cancer (KEYNOTE-012): a non-randomised, openlabel, phase 1b study," The Lancet Oncology, vol. 18, no. 2, pp. 212-220, 2017.

[34] A. V. Balar, D. E. Castellano, P. H. O’Donnell et al., "Pembrolizumab as first-line therapy in cisplatin-ineligible advanced urothelial cancer: results from the total KEYNOTE-052 study population," Journal of Clinical Oncology, vol. 35, supplement 6S, 2017, abstract 284.

[35] A. Balar, J. Bellmunt, P. O’Donnell et al., "Pembrolizumab (pembro) as first-line therapy for advanced/unresectable or metastatic urothelial cancer: preliminary results from the phase 2 KEYNOTE-052 study," Annals of Oncology, vol. 27, no. 6, pp. 1-36, 2016.

[36] J. Bellmunt, R. de Wit, D. J. Vaughn et al., "Pembrolizumab as second-line therapy for advanced urothelial carcinoma," New England Journal of Medicine, vol. 376, no. 11, pp. 1015-1026, 2017.

[37] B. C. Carthon, J. D. Wolchok, J. Yuan et al., "Preoperative CTLA4 blockade: tolerability and immune monitoring in the setting of a presurgical clinical trial," Clinical Cancer Research, vol. 16, no. 10, pp. 2861-2871, 2010.

[38] M. N. Fishman, D. A. Vaena, and P. Singh, "Phase Ib/II study of an IL-2/T-cell receptor fusion protein in combination with gemcitabine and cisplatin in advanced or metastatic chemorefractory urothelial cancer (UC)," Journal of Clinical Oncology, vol. 33, 2015, abstract 4515.

[39] D. Wu, Z. Zhang, H. Pan, Y. Fan, P. Qu, and J. Zhou, "Upregulation of the B7/CD28 family member B7-H3 in bladder cancer," Oncology Letters, vol. 9, no. 3, pp. 1420-1424, 2015.

[40] J. Powderly, G. Cote, K. Flaherty et al., "Interim results of an ongoing phase 1, dose escalation study of mga271 (enoblituzumab), an fc-optimized humanized anti-b7-h3 monoclonal antibody, in patients with advanced solid cancer," Presented at 2015 Society for Immunotherapy of Cancer (SITC) Annual Meeting.

[41] M. Croft, T. So, W. Duan, and P. Soroosh, "The significance of OX40 and OX40L to T-cell biology and immune disease," Immunological Reviews, vol. 229, no. 1, pp. 173-191, 2009.

[42] T. H. Watts, "TNF/TNFR family members in costimulation of T cell responses," Annual Review of Immunology, vol. 23, pp. 2368, 2005.

[43] A. Sherif, M. N. Hasan, P. Marits, M. Karlsson, O. Winqvist, and M. Thörn, "Feasibility of T-cell-based adoptive immunotherapy in the first 12 patients with advanced urothelial urinary bladder cancer. Preliminary data on a new immunologic treatment based on the sentinel node concept," European Urology, vol. 58, no. 1, pp. 105-111, 2010.

[44] S. Oudard, S. Culine, Y. Vano et al., "Multicentre randomised phase II trial of gemcitabine + platinum, with or without trastuzumab, in advanced or metastatic urothelial carcinoma overexpressing Her2," European Journal of Cancer, vol. 51, no. 1, pp. 45-54, 2015.

[45] A. H. Bryce, R. Kurzrock, F. Meric-Bernstam et al., "Pertuzumab plus trastuzumab for HER2-positive metastatic urothelial cancer (mUC): preliminary data from MyPathway," Journal of Clinical Oncology, vol. 35, supplement 6S, 2017, abstract 348.
[46] S. J. Brancato, K. Lewi, and P. K. Agarwal, "Evolving immunotherapy strategies in urothelial cancer," American Society of Clinical Oncolology, pp. e284-e290, 2015, Educational Book.

[47] M. A. Morse, R. Chapman, J. Powderly et al., "Phase I study utilizing a novel antigen-presenting cell-targeted vaccine with toll-like receptor stimulation to induce immunity to selfantigens in cancer patients," Clinical Cancer Research, vol. 17, no. 14, pp. 4844-4853, 2011.

[48] N. Makise, T. Morikawa, T. Nakagawa et al., "MAGE-A expression, immune microenvironment, and prognosis in upper urinary tract carcinoma," Human Pathology, vol. 50, pp. 62-69, 2016.

[49] T. Nishiyama, M. Tachibana, Y. Horiguchi et al., "Immunotherapy of bladder cancer using autologous dendritic cells pulsed with human lymphocyte antigen-a24-specific MAGE-3 peptide," Clinical Cancer Research, vol. 7, no. 1, pp. 23-31, 2001.

[50] M. Marchand, C. J. A. Punt, S. Aamdal et al., "Immunisation of metastatic cancer patients with MAGE-3 protein combined with adjuvant SBAS-2: a clinical report," European Journal of Cancer, vol. 39, no. 1, pp. 70-77, 2003.

[51] R. Wang and H. Y. Wang, "Immune targets and neoantigens for cancer immunotherapy and precision medicine," Cell Research, vol. 27, no. 1, pp. 11-37, 2016.

[52] M. Noguchi, K. Matsumoto, H. Uemura et al., "An open-label, randomized phase II trial of personalized peptide vaccination in patients with bladder cancer that progressed after platinumbased chemotherapy," Clinical Cancer Research, vol. 22, no. 1, pp. 54-60, 2016.

[53] X. Meng, Z. Huang, F. Teng, L. Xing, and J. Yu, "Predictive biomarkers in PD-1/PD-L1 checkpoint blockade immunotherapy," Cancer Treatment Reviews, vol. 41, no. 10, pp. 868-876, 2015.

[54] S. Champiat, O. Lambotte, E. Barreau et al., "Management of immune checkpoint blockade dysimmune toxicities: a collaborative position paper," Annals of Oncology, vol. 27, no. 4, Article ID mdv623, pp. 559-574, 2016.

[55] P. A. Ascierto, M. Del Vecchio, C. Robert et al., "Ipilimumab 10 $\mathrm{mg} / \mathrm{kg}$ versus ipilimumab $3 \mathrm{mg} / \mathrm{kg}$ in patients with unresectable or metastatic melanoma: a randomised, double-blind, multicentre, phase 3 trial," The Lancet Oncology, vol. 18, no. 5, pp. 611622, 2017. 


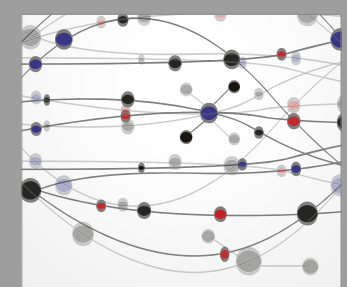

The Scientific World Journal
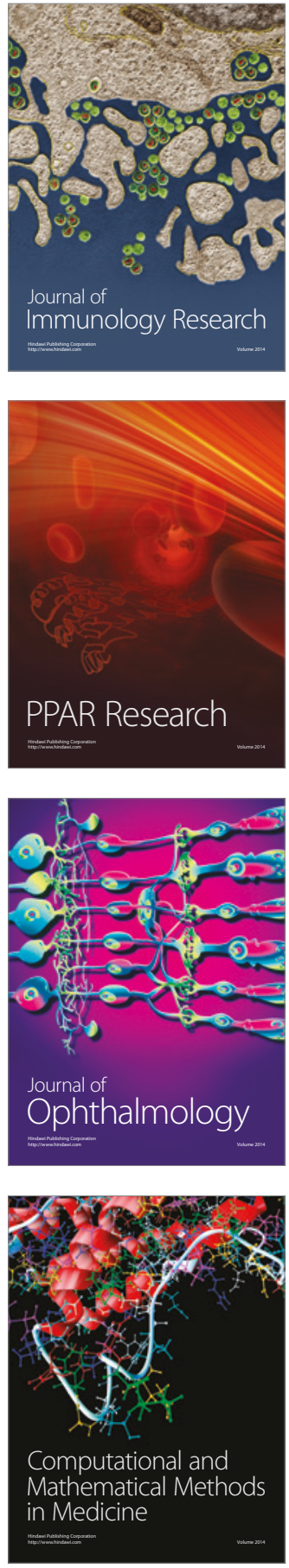

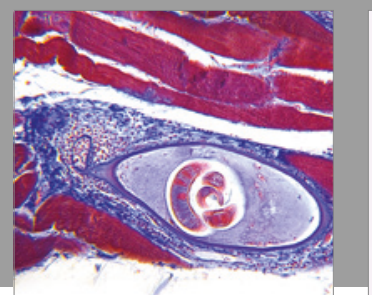

Gastroenterology Research and Practice
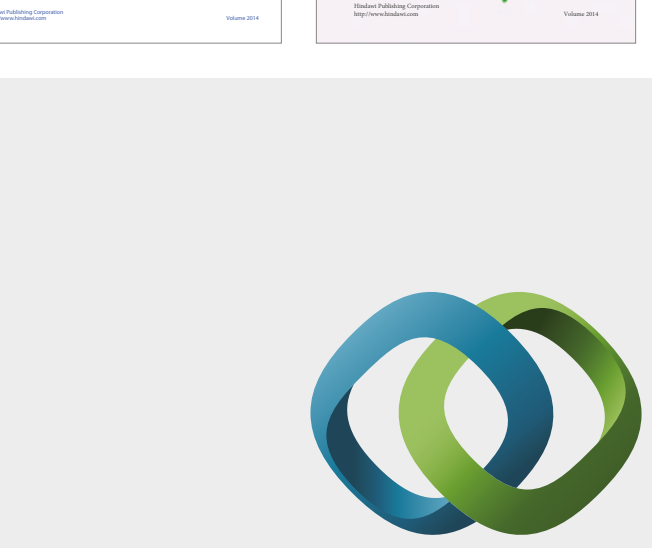

\section{Hindawi}

Submit your manuscripts at

https://www.hindawi.com
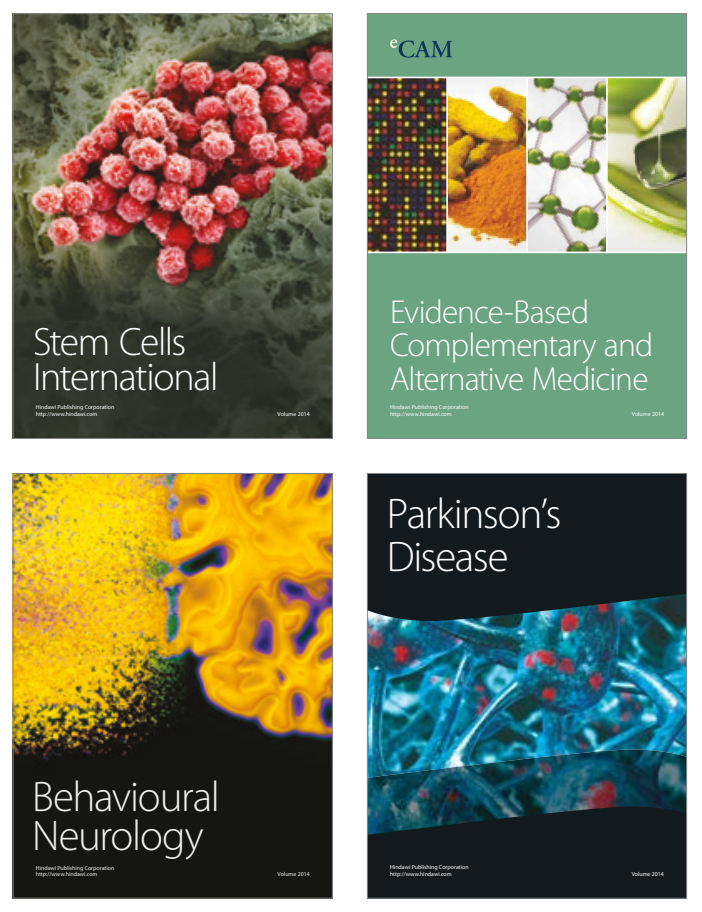
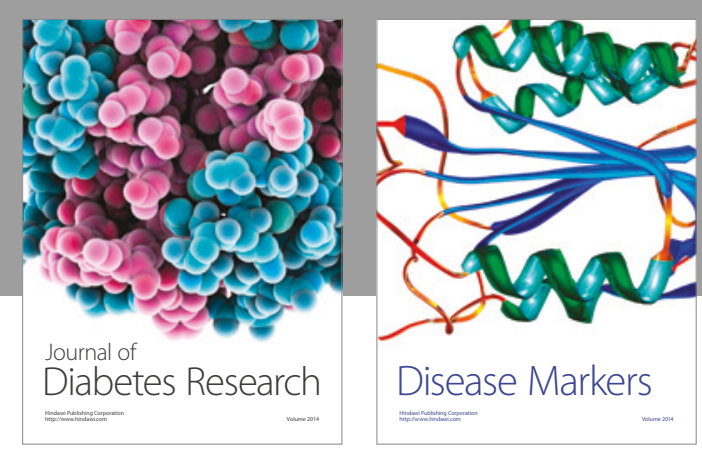

Disease Markers
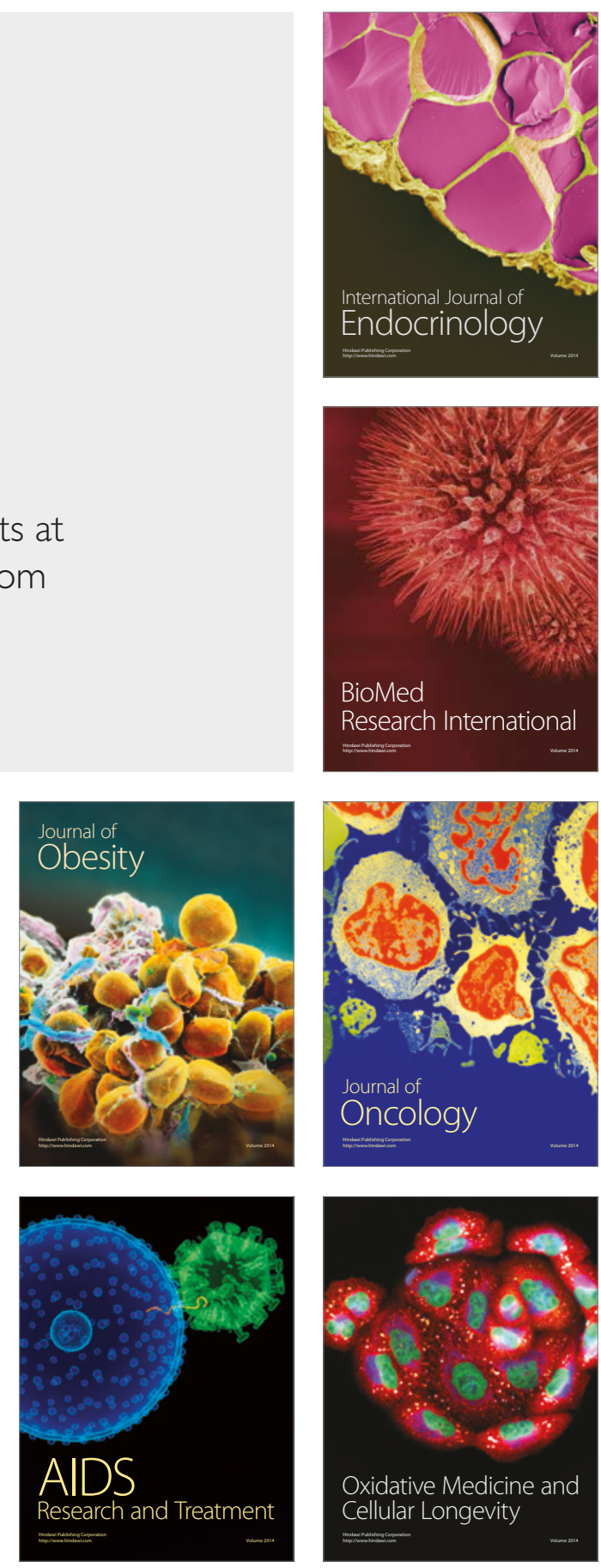